\title{
EFFECTS OF ACUTE ANOXIA ON THE CIRCULATION AND RESPIRATION IN PATIENTS WITH CHRONIC PUL- MONARY DISEASE STUDIED DURING THE "STEADY STATE" 1
}

\author{
By A. P. FISHMAN, ${ }^{2}$ J. McClEMENT, 3 A. HIMMELSTEIN, ANd A. COURNAND \\ (From the Department of Medicine, Columbia University, College of Physicians and Surgeons, \\ and the Cardio-Pulmonary Laboratory of the First Medical and Chest Services \\ [Columbia University Division], Bellevue Hospital, New York, N. Y.)
}

(Submitted for publication May 10, 1952; accepted June 13, 1952)

\section{INTRODUCTION}

The effect of acutely induced anoxia upon the respiration and circulation of man and animals has been repeatedly investigated $(1-4)$. Various circulatory responses have been described, and it is apparent that the observations of different investigators have sometimes been divergent. For example, following the induction of acute anoxia, the minute output of the heart has been noted by individual authors to increase, decrease, or remain unchanged. Grollman (1) reviewed these results and ascribed them to several readily discernible causes: 1 ) inaccuracies inherent in the methods used to measure cardiac output; 2) failure to distinguish between the physiologic responses of anesthetized versus unanesthetized animals ; 3) the variety of species studied, and 4) dissimilar degrees of anoxia.

A previous report from this laboratory on the effects of acute anoxia on pulmonary artery pressure (3) included measurements of the cardiac output using the "Direct Fick Method" in five normal subjects. It was found in these studies that short periods of anoxia (breathing 10 per cent oxygen for approximately 10 minutes) usually resulted in a decrease of the estimated cardiac output. This response to anoxia which has been observed in normal individuals, merits further consideration in patients with chronic pulmonary disease where spontaneous variations in anoxia

\footnotetext{
1 This investigation was supported (in part) by a research grant (PHS Grant $\mathrm{H}-833[\mathrm{C}]$ ) from the $\mathrm{Na}$ tional Heart Institute of the National Institutes of Health, Public Health Service, with additional support from the Life Insurance Medical Research Fund and the American Heart Association.

2 Established Investigator of the American Heart Association.

3 James Alexander Miller Research Fellow.
}

and cardiac output incident to their daily activity may contribute to the evolution of cardiopulmonary disease.

It is the purpose of this report to analyze the effects of breathing gas mixtures with various oxygen concentrations upon the circulation of 35 patients with chronic pulmonary disease. Because a "steady state" of the respiration and circulation is essential when one measures cardiac output during cardiac catheterization by the Fick principle, a special attempt has been made to include only studies in which such a state was attained and maintained during the period of observation.

\section{METHODS}

All patients were studied in the unanesthetized, postabsorptive, "basal" state. The observations were usually begun approximately one-half hour after arrival in the laboratory, and considerable attention was given to the achievement of complete relaxation during the period of observation. This was facilitated by respiratory measurements and arterial puncture on previous days which served to familiarize the patient with the laboratory, its apparatus and personnel. In some, cardiac catheterization had been previously performed. All determinations were completed within two hours after placement of the cardiac catheter. The methods used were identical with those previously described from this laboratory $(5,6)$.

The heart rate was observed on the electrocardiogram throughout the entire procedure, including the period of cardiac catheterization and arterial puncture. Lability of heart rate while the subject was at rest and breathing ambient air, supplemented other clinical guides to sympathetic overactivity (tachypnea, moist skin, wide pupils).

The initial series of determinations of cardiac output and blood pressures (pulmonary artery and brachial artery) were made while the relaxed subject was breathing 21 per cent oxygen. After expired gas, mixed venous blood and arterial blood had been simultaneously collected to measure the cardiac output, the entire procedure was repeated with the subject inspiring a higher (if original 
arterial oxygen saturation was less than 90 per cent), or lower oxygen mixture. The blood and expired gas samples were also used to calculate the alveolar-arterial (A-A) oxygen gradient. All determinations were required to check in duplicate. The techniques used are detailed elsewhere (6). The $\mathrm{pO}_{2}$ of mixed venous blood was obtained by plotting oxygen content (Van SlykeNeill) on a standard oxyhemoglobin dissociation curve using the corresponding $\mathrm{pH}$ determined by the MacInnes-Belcher glass electrode. The oxygen and carbon dioxide tensions of arterial blood were determined directly (7), and the latter was also estimated from the line charts of Van Slyke and Sendroy, using the $\mathrm{CO}_{2}$ content and the $\mathrm{pH}$ of the whole blood. Systolic and diastolic pressures were calculated as averages of two complete respiratory cycles; the mean pressure was obtained by planimetric integration of the area included within these boundaries. Variations were considered significant only if they exceeded limits previously defined in this laboratory (8-10) and include deviations greater than $\pm 5 \mathrm{~mm}$. $\mathrm{Hg}$ for pulmonary artery mean pressure and -12 to $+18 \mathrm{~mm}$. $\mathrm{Hg}$ for systemic artery mean pressure. Similarly under the conditions of these experiments, any cardiac output change exceeding 9 per cent of control was considered significant.

The inspired gas mixtures used in these studies included 100 per cent oxygen and mixtures of $33,25,21$, $18,16,14$, and 12 per cent oxygen in nitrogen, administered through demand-type valves or from an anesthesia bag. At least two experiments, each at a different level of arterial oxygen saturation, were completed for each subject. In the following pages, "low oxygen" refers to inspired gas mixtures containing less than 21 per cent oxygen; conversely, "high oxygen" mixtures contain more than 21 per cent oxygen. The specific concentrations used are indicated in the tables.

\section{Criteria used for defining the "steady state"}

Three criteria were used as guides to stability of the respiration and circulation (the "steady state") as successive levels of oxygenation. These were 1) emotional stability, 2) constancy of oxygen consumption, and 3) constancy of the respiratory exchange ratio (RQ). Significant deviations in any of these three criteria served to exclude a given subject from this study.

It is known that clinical manifestations of sympathetic overactivity are at best a crude guide to emotional disturbance. However, in response to emotional stimulation, oxygen consumption nearly always increases (11); in absence of such stimulation, oxygen consumption remains remarkably constant from day to day when determined under comparable conditions $(12,13)$. Neither acute anoxia per se nor the hyperventilation induced by it (14-16) causes increase of oxygen consumption. Because of these considerations and in keeping with a large body of data previously gathered in this laboratory during cardiac catheterization (8), any change in oxygen consumption which exceeded +11 per cent eliminated the experiment from inclusion in this study.
If the oxygen consumption is constant (within the limits defined above) at the two levels of oxygenation, an elevation in respiratory gas exchange ratio (R.Q.) becomes a reflection of respiratory rather than metabolic carbon dioxide liberation (unstable respiratory state). A review of a series of patients previously studied under comparable conditions in this laboratory (8) indicated that all of 20 patients had R.Q. values less than 1.0 during repeated determinations, and that 17 of the 20 deviated from the initial value by less than 0.13 during the second determination performed within one hour. In selecting the group to be presented below, any patient with pulmonary disease with any R.Q. greater than 1.0, or with a second R.Q. which differed from the first by more than 0.11 , was excluded.

\section{RESULTS}

These data were derived from 45 complete studies on 35 subjects with pulmonary disease. The clinical diagnoses, physical findings, procedures and data concerning the steady state are indicated in Table I.

\section{A. Ventilation}

With three exceptions all of these subjects, while breathing ambient air, had a higher than normal respiratory minute volume (Table I). This observation is in accord with similar respiratory studies previously done on the same patients in this laboratory. In the experiments employing high oxygen, there was no significant change in ventilation. However, during low oxygen breathing, the volume of ventilation usually exceeded control levels.

\section{B. Carbon dioxide tension in arterial blood (Table I)}

Seventeen of 35 patients, while breathing ambient air, had normal $(39.4 \pm 2.8)$ arterial $\mathrm{pCO}_{2}$. Ten had low $\mathrm{pCO}_{2}$ values, probably secondary to their chronic hyperventilation. Eight, however, had elevated arterial $\mathrm{pCO}_{2}$, the two highest values occurring in two subjects with emphysema and cor pulmonale (Cases 32 and 33). Following exposure to low oxygen, the arterial $\mathrm{pCO}_{2}$ was usually lower than on ambient air. The arterial $\mathrm{pCO}_{2}$ while the subjects were breathing a high oxygen mixture showed no consistent direction of change.

\section{Heart rate}

Resting heart rates ranged from 48 to 137 . Exposure to high oxygen usually caused slowing or 
TABLE I

Physical characteristics, clinical diagnoses, and orienting data in 35 patients studied at successive levels of oxygenation

\begin{tabular}{|c|c|c|c|c|c|c|c|c|c|}
\hline Case & Sex & Age & $\underset{m^{2}}{\text { BSA }}$ & Diagnosis & \begin{tabular}{|c|} 
Concen- \\
tration \\
inspired \\
oxygen \\
oolumes \%
\end{tabular} & \begin{tabular}{|c|}
$\begin{array}{c}\text { Ventila- } \\
\text { tion } \\
\text { tiit. } / \mathrm{min}^{2} / \mathrm{m}^{2}\end{array}$ \\
\end{tabular} & R.Q. & \begin{tabular}{|c|} 
Oxygen \\
consump \\
tion - \\
c. $/$ min. $^{2} / \mathrm{m}^{2}$
\end{tabular} & $\begin{array}{c}\text { Arterial } \\
\mathrm{pCO} \\
\mathrm{mm} . \mathrm{H}_{8}\end{array}$ \\
\hline \multicolumn{10}{|c|}{ (a) Normal and low oxygen } \\
\hline 1. G. W. & $\mathbf{f}$ & 38 & 1.67 & $\begin{array}{l}\text { Bronchiectasis; interventricular sep- } \\
\text { tal defect }\end{array}$ & $\begin{array}{l}21 \\
10\end{array}$ & $\begin{array}{l}6.6 \\
8.3\end{array}$ & $\begin{array}{l}.87 \\
.82\end{array}$ & $\begin{array}{l}113 \\
124\end{array}$ & $\begin{array}{l}40 \\
31\end{array}$ \\
\hline 2. L. K. & $\mathbf{m}$ & 29 & 1.80 & $\begin{array}{l}\text { Diffuse pulmonary infiltration, cause } \\
\text { unknown }\end{array}$ & $\begin{array}{l}21 \\
12\end{array}$ & $\begin{array}{l}5.1 \\
7.2\end{array}$ & $\begin{array}{l}.86 \\
.97\end{array}$ & $\begin{array}{l}174 \\
177\end{array}$ & $\begin{array}{l}34 \\
29\end{array}$ \\
\hline 3. M. Mc & $\mathbf{f}$ & 22 & 1.42 & $\begin{array}{l}\text { Diffuse pulmonary fibrosis, sclero- } \\
\text { derma }\end{array}$ & $\begin{array}{l}21.5 \\
14\end{array}$ & $\begin{array}{l}5.1 \\
4.8\end{array}$ & $\begin{array}{l}.85 \\
.85\end{array}$ & $\begin{array}{l}133 \\
132\end{array}$ & $\begin{array}{l}42 \\
37\end{array}$ \\
\hline 4. J. L. & $\mathbf{m}$ & 49 & 1.90 & Silicosis; chr. pulm. emphysema & $\begin{array}{l}21 \\
12\end{array}$ & $\begin{array}{l}3.8 \\
5.2\end{array}$ & $\begin{array}{l}.87 \\
.91\end{array}$ & $\begin{array}{l}134 \\
148\end{array}$ & $\begin{array}{l}36 \\
36\end{array}$ \\
\hline 5. D. P. & $\mathbf{m}$ & 36 & 1.75 & $\begin{array}{l}\text { Bronchiectasis; diffuse pulmonary in- } \\
\text { filtration }\end{array}$ & $\begin{array}{l}21.5 \\
18.5\end{array}$ & $\begin{array}{l}3.5 \\
3.9\end{array}$ & $\begin{array}{l}.73 \\
.79\end{array}$ & $\begin{array}{l}127 \\
135\end{array}$ & $\begin{array}{l}41 \\
38\end{array}$ \\
\hline 6. J. R. & $\mathbf{m}$ & 40 & 1.38 & Acute hematogenous tuberculosis & $\begin{array}{l}21 \\
16 \\
21.5 \\
16\end{array}$ & $\begin{array}{l}4.4 \\
4.2 \\
4.4 \\
5.2\end{array}$ & $\begin{array}{l}.83 \\
.94 \\
.86 \\
.84\end{array}$ & $\begin{array}{l}112 \\
102 \\
124 \\
128\end{array}$ & $\begin{array}{l}40 \\
37 \\
37 \\
36\end{array}$ \\
\hline 7. T. Me & f & 30 & 1.59 & Chr. pulm. tuberculosis & $\begin{array}{l}21 \\
16\end{array}$ & $\begin{array}{l}4.8 \\
5.6\end{array}$ & $\begin{array}{l}.86 \\
.86\end{array}$ & $\begin{array}{l}129 \\
141\end{array}$ & $\begin{array}{l}43 \\
40\end{array}$ \\
\hline 8. E. H. & $\mathbf{m}$ & 17 & 1.47 & $\begin{array}{l}\text { Severe diffuse pulmonary granulo- } \\
\text { mata, cause unknown }\end{array}$ & $\begin{array}{l}21 \\
16 \\
21.5 \\
16\end{array}$ & $\begin{array}{l}8.3 \\
8.9 \\
7.6 \\
7.0\end{array}$ & $\begin{array}{l}.86 \\
.83 \\
.95 \\
.96\end{array}$ & $\begin{array}{l}197 \\
199 \\
172 \\
170\end{array}$ & $\begin{array}{l}40 \\
34 \\
32 \\
31\end{array}$ \\
\hline 9. S. A. & $\mathbf{m}$ & 60 & 1.65 & $\begin{array}{l}\text { Bronchiectasis; chr. pulm. emphy- } \\
\text { sema }\end{array}$ & $\begin{array}{l}21 \\
16 \\
14\end{array}$ & $\begin{array}{l}6.4 \\
5.8 \\
5.6\end{array}$ & $\begin{array}{l}.94 \\
.98 \\
.97\end{array}$ & $\begin{array}{l}123 \\
128 \\
125\end{array}$ & $\begin{array}{l}41 \\
38 \\
39\end{array}$ \\
\hline 10. Q. G. & f & 43 & 1.66 & $\begin{array}{l}\text { Severe diffuse pulmonary granulo- } \\
\text { mata, Boeck's sarcoid }\end{array}$ & $\begin{array}{l}21.5 \\
17 \\
21 \\
17\end{array}$ & $\begin{array}{l}6.5 \\
7.2 \\
6.3 \\
6.8\end{array}$ & $\begin{array}{l}.76 \\
.85 \\
.88 \\
.94\end{array}$ & $\begin{array}{l}146 \\
141 \\
157 \\
145\end{array}$ & $\begin{array}{l}39 \\
37 \\
39 \\
35\end{array}$ \\
\hline 11. C. D. & $\mathbf{m}$ & 23 & 1.67 & Chr. pulm. tuberculosis & $\begin{array}{l}21 \\
16\end{array}$ & $\begin{array}{l}4.8 \\
5.2\end{array}$ & $\begin{array}{l}.72 \\
.80\end{array}$ & $\begin{array}{l}157 \\
146\end{array}$ & $\begin{array}{l}34 \\
32\end{array}$ \\
\hline 12. H. K. & f & 25 & 1.53 & Bronchiectasis; bilateral lobectomy & $\begin{array}{l}21 \\
16\end{array}$ & $\begin{array}{l}5.0 \\
5.0\end{array}$ & $\begin{array}{l}.79 \\
.84\end{array}$ & $\begin{array}{l}146 \\
135\end{array}$ & $\begin{array}{l}34 \\
34\end{array}$ \\
\hline 13. E. C. & $\mathbf{m}$ & 29 & 1.69 & Chr. pulm. tuberculosis & $\begin{array}{l}21 \\
16\end{array}$ & $\begin{array}{l}5.6 \\
5.4\end{array}$ & $\begin{array}{l}.82 \\
.88\end{array}$ & $\begin{array}{l}125 \\
120\end{array}$ & $\begin{array}{l}39 \\
36\end{array}$ \\
\hline 14. K. C. & f & 31 & 1.73 & Acute hematogenous tuberculosis & $\begin{array}{l}21 \\
16\end{array}$ & $\begin{array}{l}3.7 \\
3.8\end{array}$ & $\begin{array}{l}.82 \\
.90\end{array}$ & $\begin{array}{l}112 \\
104\end{array}$ & $\begin{array}{l}38 \\
40\end{array}$ \\
\hline 15. W. H. & $\mathbf{m}$ & 22 & 1.90 & $\begin{array}{l}\text { Diffuse pulmonary granulomata, } \\
\text { Boeck's sarcoid }\end{array}$ & $\begin{array}{l}21 \\
16\end{array}$ & $\begin{array}{l}4.7 \\
6.5\end{array}$ & $\begin{array}{l}.78 \\
.89\end{array}$ & $\begin{array}{l}169 \\
167\end{array}$ & $\begin{array}{l}35 \\
34\end{array}$ \\
\hline 16. F. A. & $\mathbf{m}$ & 48 & 1.55 & $\begin{array}{l}\text { Chr. pulm. tuberculosis; acute hema- } \\
\text { togenous tuberculosis }\end{array}$ & $\begin{array}{l}21 \\
16\end{array}$ & $\begin{array}{l}7.4 \\
7.4\end{array}$ & $\begin{array}{l}.85 \\
.83\end{array}$ & $\begin{array}{l}147 \\
148\end{array}$ & $\begin{array}{l}35 \\
35\end{array}$ \\
\hline 17. F. L. & $\mathbf{m}$ & 23 & 1.85 & $\begin{array}{l}\text { Severe diffuse pulmonary fibrosis, } \\
\text { beryllium exposure }\end{array}$ & $\begin{array}{l}21 \\
16\end{array}$ & $\begin{array}{l}5.8 \\
6.9\end{array}$ & $\begin{array}{l}.78 \\
.88\end{array}$ & $\begin{array}{l}140 \\
131\end{array}$ & $\begin{array}{l}37 \\
35\end{array}$ \\
\hline 18. R. S. & $\mathbf{m}$ & 25 & 1.85 & $\begin{array}{l}\text { Moderate diffuse pulmonary granu- } \\
\text { lomata, Boeck's sarcoid }\end{array}$ & $\begin{array}{l}21 \\
16\end{array}$ & $\begin{array}{l}4.3 \\
4.7\end{array}$ & $\begin{array}{l}.83 \\
.92\end{array}$ & $\begin{array}{l}149 \\
146\end{array}$ & $\begin{array}{l}39 \\
36\end{array}$ \\
\hline 19. N. I. & $\mathbf{m}$ & 31 & 1.35 & $\begin{array}{l}\text { Moderate diffuse pulmonary granu- } \\
\text { lomata, Boeck's sarcoid }\end{array}$ & $\begin{array}{l}21 \\
16\end{array}$ & $\begin{array}{l}4.4 \\
5.4\end{array}$ & $\begin{array}{l}.82 \\
.91\end{array}$ & $\begin{array}{l}121 \\
128\end{array}$ & $\begin{array}{l}34 \\
35\end{array}$ \\
\hline 20. C. L. & f & 21 & 1.74 & $\begin{array}{l}\text { Moderate diffuse pulmonary granulo- } \\
\text { mata, Boeck's sarcoid }\end{array}$ & $\begin{array}{l}21 \\
16\end{array}$ & $\begin{array}{l}7.0 \\
7.9\end{array}$ & $\begin{array}{l}.81 \\
.82\end{array}$ & $\begin{array}{l}166 \\
168\end{array}$ & $\begin{array}{l}45 \\
43\end{array}$ \\
\hline
\end{tabular}


TABLE I.-Continued

\begin{tabular}{|c|c|c|c|c|c|c|c|c|c|}
\hline Case & Sex & Age & $\underset{m^{2}}{\mathbf{B S A}}$ & Diagnosis & $\begin{array}{l}\text { Concen- } \\
\text { tration } \\
\text { inspired } \\
\text { oxygen } \\
\text { volumes \% }\end{array}$ & $\begin{array}{c}\text { Ventila- } \\
\text { tion } \\
\text { lit. } / \text { min. }^{\prime} / \mathrm{m}^{2}\end{array}$ & R.Q. & $\begin{array}{c}\text { Oxygen } \\
\text { consump- } \\
\text { tion } \\
c c . / \mathrm{min}^{2} / \mathrm{m}^{2}\end{array}$ & $\begin{array}{c}\text { Arterial } \\
\mathrm{pCO}_{\mathbf{2}} \\
\mathbf{m} . \mathrm{Hg}_{\boldsymbol{g}}\end{array}$ \\
\hline
\end{tabular}

(a) Normal and low oxygen-Continued

\begin{tabular}{l|l|l|l|l|l|l|l|l|l|l|l}
\hline 21. J. R. & $\mathrm{m}$ & 23 & 1.57 & $\begin{array}{l}\text { Acute hematogenous tuberculosis; 11 } \\
\text { days after start Rx with strepto- } \\
\text { mycin }\end{array}$ & $\begin{array}{l}21.5 \\
16\end{array}$ & $\begin{array}{l}5.5 \\
5.7\end{array}$ & $\begin{array}{l}.79 \\
.86\end{array}$ & $\begin{array}{l}147 \\
140\end{array}$ & $\begin{array}{l}40 \\
36\end{array}$ \\
\hline
\end{tabular}

(b) Normal, low and high oxygen

\begin{tabular}{|c|c|c|c|c|c|c|c|c|c|}
\hline 22. B. B. & $\mathbf{m}$ & 52 & 1.54 & $\begin{array}{l}\text { Bronchial asthma; chr. pulm. emphy- } \\
\text { sema; cor pulmonale, not in failure }\end{array}$ & $\begin{array}{l}21 \\
16 \\
21 \\
25\end{array}$ & $\begin{array}{l}6.6 \\
7.2 \\
7.3 \\
6.7\end{array}$ & $\begin{array}{c}.84 \\
.90 \\
1.0 \\
.90\end{array}$ & $\begin{array}{l}137 \\
151 \\
150 \\
146\end{array}$ & $\begin{array}{l}47 \\
42 \\
41 \\
39\end{array}$ \\
\hline 23. A. P. & $\mathbf{m}$ & 49 & 1.55 & $\begin{array}{l}\text { Chr. pulm. emphysema; cor pul- } \\
\text { monale, in failure }\end{array}$ & $\begin{array}{l}21.5 \\
16 \\
21 \\
30\end{array}$ & $\begin{array}{l}5.8 \\
6.5 \\
5.4 \\
5.9\end{array}$ & $\begin{array}{l}.88 \\
.97 \\
.84 \\
.93\end{array}$ & $\begin{array}{l}141 \\
144 \\
127 \\
128\end{array}$ & $\begin{array}{l}45 \\
46 \\
42 \\
42\end{array}$ \\
\hline 24. D. Mc & $\mathbf{m}$ & 62 & 1.61 & $\begin{array}{l}\text { Carcinoma of bronchus, RML; chr. } \\
\text { pulm. emphysema }\end{array}$ & $\begin{array}{l}21 \\
16 \\
21 \\
25\end{array}$ & $\begin{array}{l}5.7 \\
6.5 \\
5.7 \\
6.1\end{array}$ & $\begin{array}{l}.80 \\
.88 \\
.80 \\
.83\end{array}$ & $\begin{array}{l}149 \\
143 \\
149 \\
138\end{array}$ & $\begin{array}{l}39 \\
39 \\
39 \\
42\end{array}$ \\
\hline 25. P. B. & f & 42 & 1.56 & $\begin{array}{l}\text { Severe diffuse pulmonary granulo- } \\
\text { mata, Boeck's sarcoid }\end{array}$ & $\begin{array}{l}12.5 \\
17.4 \\
21 \\
25\end{array}$ & $\begin{array}{l}7.4 \\
8.0 \\
7.4 \\
8.3\end{array}$ & $\begin{array}{l}.92 \\
.96 \\
.92 \\
.98\end{array}$ & $\begin{array}{l}156 \\
157 \\
156 \\
164\end{array}$ & $\begin{array}{l}40 \\
36 \\
40 \\
40\end{array}$ \\
\hline 26. W. B. & $\mathbf{m}$ & 46 & 1.61 & $\begin{array}{l}\text { Bronchial asthma; chr. pulm. emphy- } \\
\text { sema; cor pulmonale, not in failure }\end{array}$ & $\begin{array}{l}21.5 \\
16 \\
21 \\
30\end{array}$ & $\begin{array}{l}5.8 \\
6.0 \\
5.8 \\
5.4\end{array}$ & $\begin{array}{l}.85 \\
.87 \\
.85 \\
.83\end{array}$ & $\begin{array}{l}138 \\
138 \\
138 \\
135\end{array}$ & $\begin{array}{l}38 \\
36 \\
40 \\
43\end{array}$ \\
\hline 27. P. B. & $\mathbf{m}$ & 36 & 1.49 & $\begin{array}{l}\text { Severe diffuse pulmonary granulo- } \\
\text { mata, Boeck's sarcoid }\end{array}$ & $\begin{array}{l}21 \\
17.4 \\
21 \\
25\end{array}$ & $\begin{array}{l}8.8 \\
8.8 \\
8.8 \\
8.8\end{array}$ & $\begin{array}{l}.77 \\
.83 \\
.77 \\
.82\end{array}$ & $\begin{array}{l}203 \\
184 \\
205 \\
187\end{array}$ & $\begin{array}{l}35 \\
32 \\
37 \\
36\end{array}$ \\
\hline 28. M. L. & f & 29 & 1.32 & $\begin{array}{l}\text { Severe diffuse pulmonary infiltration, } \\
\text { cause unknown }\end{array}$ & $\begin{array}{l}21 \\
17 \\
21 \\
25\end{array}$ & $\begin{array}{l}7.3 \\
7.3 \\
6.4 \\
6.5\end{array}$ & $\begin{array}{l}.77 \\
.84 \\
.70 \\
.80\end{array}$ & $\begin{array}{l}147 \\
141 \\
153 \\
141\end{array}$ & $\begin{array}{l}29 \\
28 \\
37 \\
39\end{array}$ \\
\hline
\end{tabular}

(c) Normal and high oxygen

\begin{tabular}{|c|c|c|c|c|c|c|c|c|c|}
\hline 29. J.P. & $\mathbf{m}$ & 43 & 1.66 & $\begin{array}{l}\text { Bronchiectasis; cor pulmonale, in fail- } \\
\text { ure }\end{array}$ & $\begin{array}{r}21 \\
100\end{array}$ & 6.9 & .75 & $\begin{array}{l}182 \\
206\end{array}$ & $\frac{42}{-}$ \\
\hline 30. G. Na & $\mathbf{f}$ & 52 & 1.87 & $\begin{array}{l}\text { Bronchial asthma; chr. pulm. emphy- } \\
\text { sema }\end{array}$ & 21.5 & $\begin{array}{l}5.9 \\
6.6\end{array}$ & $\begin{array}{l}.72 \\
.74\end{array}$ & $\begin{array}{l}150 \\
140\end{array}$ & $\begin{array}{l}52 \\
54\end{array}$ \\
\hline 31. P. L. & $\mathbf{m}$ & 65 & 1.60 & $\begin{array}{l}\text { Bronchial asthma; chr. pulm. emphy- } \\
\text { sema }\end{array}$ & $\begin{array}{l}21.5 \\
25 \\
33\end{array}$ & $\begin{array}{l}6.8 \\
6.7 \\
6.8\end{array}$ & $\begin{array}{l}.72 \\
.83 \\
.78\end{array}$ & $\begin{array}{l}141 \\
131 \\
138\end{array}$ & $\begin{array}{l}30 \\
35 \\
45\end{array}$ \\
\hline 32. A. Y. & $\mathbf{m}$ & 38 & 1.47 & $\begin{array}{l}\text { Bronchial asthma; chr. pulm. emphy- } \\
\text { sema; cor pulmonale in congestive } \\
\text { failure }\end{array}$ & $\begin{array}{l}21 \\
25\end{array}$ & $\begin{array}{l}4.8 \\
4.9\end{array}$ & $\begin{array}{l}.79 \\
.74\end{array}$ & $\begin{array}{l}157 \\
166\end{array}$ & $\begin{array}{l}62 \\
58\end{array}$ \\
\hline 33. M. C. & $\mathbf{m}$ & 55 & 1.54 & $\begin{array}{l}\text { Bronchial asthma; pulmonary fibro- } \\
\text { sis; chr. pulm. emphysema; cor pul- } \\
\text { monale after treatment }\end{array}$ & $\begin{array}{l}21 \\
25\end{array}$ & $\begin{array}{l}6.2 \\
6.1\end{array}$ & $\begin{array}{l}.73 \\
.70\end{array}$ & $\begin{array}{l}168 \\
161\end{array}$ & $\begin{array}{l}58 \\
55\end{array}$ \\
\hline 34. E. M. & f & 60 & 1.73 & $\begin{array}{l}\text { Severe diffuse pulmonary infiltration, } \\
\text { cause unknown }\end{array}$ & $\begin{array}{r}21 \\
100\end{array}$ & 5.8 & $\underline{.72}$ & $\begin{array}{l}127 \\
121\end{array}$ & 39 \\
\hline 35. M. H. & f & 64 & 1.39 & $\begin{array}{l}\text { Severe diffuse pulmonary infiltration, } \\
\text { cause unknown; cor pulmonale, not } \\
\text { in failure }\end{array}$ & $\begin{array}{l}21 \\
30\end{array}$ & $\begin{array}{r}10.0 \\
9.3\end{array}$ & $\begin{array}{l}.82 \\
.80\end{array}$ & $\begin{array}{l}144 \\
140\end{array}$ & $\begin{array}{l}43 \\
49\end{array}$ \\
\hline
\end{tabular}


TABLE II

The influence of the level of oxygenation upon the circulation in 35 patients with pulmonary disease

\begin{tabular}{|c|c|c|c|c|c|c|c|c|c|c|c|}
\hline \multirow[t]{2}{*}{ Case } & \multirow{2}{*}{$\begin{array}{c}\text { Concen- } \\
\text { tration } \\
\text { inspired } \\
\text { oxygen } \\
\text { volumes \% }\end{array}$} & \multirow{2}{*}{$\begin{array}{c}\text { Alveolar } \\
\text { pO2 } \\
m m . H g\end{array}$} & \multirow{2}{*}{$\begin{array}{c}\text { Arterial } \\
\mathrm{pO}_{2} \\
\mathbf{m} \mathbf{m} . \mathrm{Hg}_{\mathbf{g}}\end{array}$} & \multirow{2}{*}{$\begin{array}{c}\mathrm{MVB} \\
\mathrm{pO}_{2} \\
m m . H_{g}\end{array}$} & \multirow{2}{*}{$\begin{array}{c}\text { Arterio- } \\
\text { venous } \\
\text { oxygen diff.* } \\
\text { volumes \% }\end{array}$} & \multirow{2}{*}{$\begin{array}{c}\text { Cardiac } \\
\text { index } \\
l i t . / m i n . \\
B S A\end{array}$} & \multirow[t]{2}{*}{$\begin{array}{l}\text { Heart rate } \\
\text { beats } / \text { min. }\end{array}$} & \multicolumn{2}{|c|}{$\begin{array}{c}\text { Pulmonary artery } \\
\text { pressure } \\
\text { mm. } \mathbf{H g}\end{array}$} & \multicolumn{2}{|c|}{$\begin{array}{c}\text { Brachial artery } \\
\text { pressure } \\
\text { mm. } \mathbf{H g}\end{array}$} \\
\hline & & & & & & & & $s / d$ & $m$ & $s / d$ & $m$ \\
\hline \multicolumn{12}{|c|}{ (a) Normal and low oxygen } \\
\hline 1 & $\begin{array}{l}21 \\
10\end{array}$ & $\begin{array}{l}97 \\
45\end{array}$ & $\begin{array}{l}73 \\
35\end{array}$ & $\begin{array}{l}44 \\
27\end{array}$ & $\begin{array}{l}2.1 \\
2.1\end{array}$ & $\begin{array}{l}6.20 \\
6.88\end{array}$ & $\begin{array}{l}100 \\
102\end{array}$ & $\begin{array}{l}25 / 10 \\
25 / 7\end{array}$ & $\begin{array}{l}18 \\
16\end{array}$ & $\begin{array}{l}131 / 76 \\
108 / 65\end{array}$ & $\begin{array}{l}99 \\
86\end{array}$ \\
\hline 2 & $\begin{array}{l}21 \\
12\end{array}$ & $\begin{array}{r}116 \\
62\end{array}$ & $\begin{array}{r}100 \\
46\end{array}$ & $\begin{array}{l}38 \\
31\end{array}$ & $\begin{array}{l}4.6 \\
3.4\end{array}$ & $\begin{array}{l}3.78 \\
5.19\end{array}$ & $\begin{array}{l}85 \\
98\end{array}$ & $\begin{array}{l}28 / 11 \\
32 / 12\end{array}$ & $\begin{array}{l}18 \\
20\end{array}$ & $\begin{array}{l}145 / 94 \\
123 / 79\end{array}$ & $\begin{array}{r}114 \\
95\end{array}$ \\
\hline 3 & $\begin{array}{l}21.5 \\
14\end{array}$ & $\begin{array}{r}100 \\
57\end{array}$ & $\begin{array}{l}94 \\
29\end{array}$ & $\begin{array}{l}37 \\
22\end{array}$ & $\begin{array}{l}3.7 \\
1.8\end{array}$ & $\begin{array}{l}3.60 \\
7.33\end{array}$ & $\begin{array}{l}79 \\
98\end{array}$ & $\begin{array}{l}32 / 13 \\
41 / 18\end{array}$ & $\begin{array}{l}20 \\
27\end{array}$ & $\begin{array}{r}95 / 57 \\
110 / 69\end{array}$ & $\begin{array}{l}74 \\
84\end{array}$ \\
\hline 4 & $\begin{array}{l}21 \\
12\end{array}$ & $\begin{array}{r}111 \\
50\end{array}$ & $\begin{array}{l}75 \\
36\end{array}$ & $\begin{array}{l}35 \\
27\end{array}$ & $\begin{array}{l}5.5 \\
4.0\end{array}$ & $\begin{array}{l}2.43 \\
3.71\end{array}$ & $\begin{array}{l}75 \\
88\end{array}$ & $\begin{array}{l}32 / 11 \\
54 / 20\end{array}$ & $\begin{array}{l}21 \\
36\end{array}$ & $\begin{array}{l}120 / 68 \\
135 / 75\end{array}$ & $\begin{array}{r}89 \\
103\end{array}$ \\
\hline 5 & $\begin{array}{l}21.5 \\
18.5\end{array}$ & $\begin{array}{r}103 \\
89\end{array}$ & $\begin{array}{l}75 \\
68\end{array}$ & $\begin{array}{l}33 \\
31\end{array}$ & $\begin{array}{l}5.3 \\
5.1\end{array}$ & $\begin{array}{l}2.39 \\
2.65\end{array}$ & $\begin{array}{l}48 \\
55\end{array}$ & $\begin{array}{l}36 / 12 \\
41 / 12\end{array}$ & $\begin{array}{l}19 \\
24\end{array}$ & $\begin{array}{l}136 / 83 \\
134 / 80\end{array}$ & $\begin{array}{l}106 \\
106\end{array}$ \\
\hline 6 & $\begin{array}{l}21 \\
16 \\
21.5 \dagger \\
16 \dagger\end{array}$ & $\begin{array}{r}108 \\
78 \\
108 \\
69\end{array}$ & $\begin{array}{r}102 \\
70 \\
84 \\
52\end{array}$ & $\begin{array}{l}34 \\
33 \\
30 \\
27\end{array}$ & $\begin{array}{l}4.2 \\
3.5 \\
4.9 \\
4.5\end{array}$ & $\begin{array}{l}2.65 \\
2.96 \\
2.38 \\
2.67\end{array}$ & $\begin{array}{l}85 \\
88 \\
68 \\
74\end{array}$ & $\begin{array}{l}12 / 4 \\
17 / 7\end{array}$ & $\begin{array}{r}9 \\
12\end{array}$ & $\begin{array}{l}104 / 71 \\
111 / 75\end{array}$ & $\begin{array}{l}84 \\
90\end{array}$ \\
\hline 7 & $\begin{array}{l}21 \\
16\end{array}$ & $\begin{array}{r}107 \\
77\end{array}$ & $\begin{array}{l}77 \\
63\end{array}$ & $\begin{array}{l}40 \\
32\end{array}$ & $\begin{array}{l}3.6 \\
3.7\end{array}$ & $\begin{array}{l}3.68 \\
3.80\end{array}$ & $\begin{array}{l}78 \\
75\end{array}$ & $\begin{array}{l}24 / 6 \\
22 / 6\end{array}$ & $\begin{array}{l}15 \\
14\end{array}$ & $\begin{array}{l}116 / 64 \\
110 / 61\end{array}$ & $\begin{array}{l}86 \\
81\end{array}$ \\
\hline 8 & $\begin{array}{l}21 \\
16 \\
21.5 \dagger \\
16 \dagger\end{array}$ & $\begin{array}{r}107 \\
72 \\
113 \\
73\end{array}$ & $\begin{array}{l}74 \\
43 \\
94 \\
56\end{array}$ & $\begin{array}{l}36 \\
28 \\
38 \\
32\end{array}$ & $\begin{array}{l}4.1 \\
4.1 \\
3.9 \\
4.1\end{array}$ & $\begin{array}{l}4.80 \\
4.88 \\
6.95 \\
6.56\end{array}$ & $\begin{array}{r}107 \\
115 \\
90 \\
100\end{array}$ & $\begin{array}{l}34 / 18 \\
41 / 23 \\
39 / 17 \\
40 / 23\end{array}$ & $\begin{array}{l}26 \\
31 \\
27 \\
31\end{array}$ & $\begin{array}{r}98 / 64 \\
82 / 55 \\
132 / 83 \\
124 / 80\end{array}$ & $\begin{array}{r}77 \\
67 \\
106 \\
98\end{array}$ \\
\hline 9 & $\begin{array}{l}21 \\
16 \\
14\end{array}$ & $\begin{array}{r}107 \\
71 \\
58\end{array}$ & $\begin{array}{l}66 \\
47 \\
38\end{array}$ & $\begin{array}{l}34 \\
31 \\
30\end{array}$ & $\begin{array}{l}4.5 \\
4.4 \\
4.0\end{array}$ & $\begin{array}{l}2.67 \\
2.85 \\
3.05\end{array}$ & $\begin{array}{l}86 \\
88 \\
88\end{array}$ & $\begin{array}{l}25 / 12 \\
31 / 13 \\
35 / 15\end{array}$ & $\begin{array}{l}17 \\
20 \\
24\end{array}$ & $\begin{array}{l}139 / 77 \\
127 / 73 \\
127 / 80\end{array}$ & $\begin{array}{r}102 \\
92 \\
102\end{array}$ \\
\hline 10 & $\begin{array}{l}21.5 \\
17 \\
21 \dagger \\
17 \dagger\end{array}$ & $\begin{array}{r}107 \\
88 \\
99 \\
78\end{array}$ & $\begin{array}{l}56 \\
36 \\
71 \\
53\end{array}$ & $\begin{array}{l}27 \\
25 \\
30 \\
27\end{array}$ & $\begin{array}{l}4.3 \\
3.3 \\
3.7 \\
3.2\end{array}$ & $\begin{array}{l}3.39 \\
4.21 \\
4.23 \\
4.52\end{array}$ & $\begin{array}{r}94 \\
94 \\
106 \\
103\end{array}$ & $\begin{array}{l}55 / 27 \\
67 / 28 \\
46 / 21 \\
56 / 23\end{array}$ & $\begin{array}{l}37 \\
43 \\
32 \\
42\end{array}$ & $\begin{array}{l}115 / 67 \\
109 / 68 \\
113 / 67\end{array}$ & $\begin{array}{l}87 \\
85 \\
85\end{array}$ \\
\hline 11 & $\begin{array}{l}21 \\
16\end{array}$ & $\begin{array}{r}114 \\
74\end{array}$ & $\begin{array}{l}78 \\
64\end{array}$ & $\begin{array}{l}37 \\
34\end{array}$ & $\begin{array}{l}5.1 \\
4.7\end{array}$ & $\begin{array}{l}3.09 \\
3.10\end{array}$ & $\begin{array}{l}80 \\
80\end{array}$ & $\begin{array}{l}18 / 8 \\
19 / 7\end{array}$ & $\begin{array}{l}12 \\
13\end{array}$ & $\begin{array}{l}122 / 77 \\
129 / 83\end{array}$ & $\begin{array}{l}94 \\
98\end{array}$ \\
\hline 12 & $\begin{array}{l}21 \\
16\end{array}$ & $\begin{array}{r}107 \\
72\end{array}$ & $\begin{array}{l}88 \\
60\end{array}$ & $\begin{array}{l}36 \\
32\end{array}$ & $\begin{array}{l}3.5 \\
3.3\end{array}$ & $\begin{array}{l}3.90 \\
4.10\end{array}$ & $\begin{array}{l}72 \\
82\end{array}$ & $\begin{array}{l}26 / 9 \\
31 / 10\end{array}$ & $\begin{array}{l}16 \\
20\end{array}$ & $\begin{array}{l}126 / 74 \\
130 / 77\end{array}$ & $\begin{array}{r}99 \\
100\end{array}$ \\
\hline 13 & $\begin{array}{l}21 \\
16\end{array}$ & $\begin{array}{r}105 \\
64\end{array}$ & $\begin{array}{l}69 \\
52\end{array}$ & $\begin{array}{l}35 \\
32\end{array}$ & $\begin{array}{l}4.7 \\
4.3\end{array}$ & $\begin{array}{l}2.64 \\
2.80\end{array}$ & $\begin{array}{l}80 \\
80\end{array}$ & $\begin{array}{l}23 / 10 \\
25 / 11\end{array}$ & $\begin{array}{l}16 \\
18\end{array}$ & $\begin{array}{l}1.14 / 77 \\
115 / 72\end{array}$ & $\begin{array}{l}94 \\
92\end{array}$ \\
\hline 14 & $\begin{array}{l}21 \\
16\end{array}$ & $\begin{array}{r}102 \\
72\end{array}$ & $\begin{array}{l}96 \\
66\end{array}$ & $\begin{array}{l}38 \\
36\end{array}$ & $\begin{array}{l}4.5 \\
3.9\end{array}$ & $\begin{array}{l}2.49 \\
2.68\end{array}$ & $\begin{array}{l}60 \\
74\end{array}$ & $\begin{array}{l}23 / 7 \\
30 / 9\end{array}$ & $\begin{array}{l}13 \\
18\end{array}$ & $\begin{array}{l}128 / 85 \\
121 / 82\end{array}$ & $\begin{array}{l}104 \\
101\end{array}$ \\
\hline 15 & $\begin{array}{l}21 \\
16\end{array}$ & $\begin{array}{r}110 \\
75\end{array}$ & $\begin{array}{l}92 \\
75\end{array}$ & $\begin{array}{l}40 \\
36\end{array}$ & $\begin{array}{l}3.5 \\
3.0\end{array}$ & $\begin{array}{l}4.83 \\
5.58\end{array}$ & $\begin{array}{l}74 \\
78\end{array}$ & $\begin{array}{l}17 / 9 \\
19 / 10\end{array}$ & $\begin{array}{l}13 \\
14\end{array}$ & $\begin{array}{l}124 / 82 \\
116 / 80\end{array}$ & $\begin{array}{l}99 \\
96\end{array}$ \\
\hline 16 & $\begin{array}{l}21 \\
16\end{array}$ & $\begin{array}{r}109 \\
67\end{array}$ & $\begin{array}{l}84 \\
54\end{array}$ & $\begin{array}{l}32 \\
30\end{array}$ & $\begin{array}{l}4.8 \\
5.1\end{array}$ & $\begin{array}{l}3.04 \\
2.90\end{array}$ & $\begin{array}{l}80 \\
80\end{array}$ & $\begin{array}{l}24 / 8 \\
28 / 10\end{array}$ & $\begin{array}{l}15 \\
18\end{array}$ & $\begin{array}{l}118 / 77 \\
108 / 71\end{array}$ & $\begin{array}{l}95 \\
89\end{array}$ \\
\hline 17 & $\begin{array}{l}21 \\
16\end{array}$ & $\begin{array}{r}109 \\
71\end{array}$ & $\begin{array}{l}86 \\
47\end{array}$ & $\begin{array}{l}36 \\
30\end{array}$ & $\begin{array}{l}4.9 \\
4.9\end{array}$ & $\begin{array}{l}2.85 \\
2.68\end{array}$ & $\begin{array}{l}80 \\
79\end{array}$ & $\begin{array}{l}30 / 14 \\
37 / 17\end{array}$ & $\begin{array}{l}21 \\
25\end{array}$ & $\begin{array}{l}116 / 85 \\
115 / 83\end{array}$ & $\begin{array}{r}101 \\
97\end{array}$ \\
\hline 18 & $\begin{array}{l}21 \\
16\end{array}$ & $\begin{array}{r}106 \\
78\end{array}$ & $\begin{array}{l}88 \\
74\end{array}$ & $\begin{array}{l}42 \\
36\end{array}$ & $\begin{array}{l}3.8 \\
4.1\end{array}$ & $\begin{array}{l}3.92 \\
3.82\end{array}$ & $\begin{array}{l}98 \\
96\end{array}$ & $\begin{array}{l}20 / 8 \\
30 / 10\end{array}$ & $\begin{array}{l}13 \\
20\end{array}$ & $\begin{array}{l}151 / 92 \\
141 / 88\end{array}$ & $\begin{array}{l}118 \\
110\end{array}$ \\
\hline 19 & $\begin{array}{l}21 \\
16\end{array}$ & $\begin{array}{r}104 \\
83\end{array}$ & $\begin{array}{l}98 \\
82\end{array}$ & $\begin{array}{l}30 \\
28\end{array}$ & $\begin{array}{l}4.1 \\
3.8\end{array}$ & $\begin{array}{l}2.96 \\
3.37\end{array}$ & $\begin{array}{r}97 \\
105\end{array}$ & $\begin{array}{l}22 / 8 \\
22 / 9\end{array}$ & $\begin{array}{l}15 \\
16\end{array}$ & $\begin{array}{l}111 / 69 \\
113 / 67\end{array}$ & $\begin{array}{l}87 \\
86\end{array}$ \\
\hline 20 & $\begin{array}{l}21 \\
16\end{array}$ & $\begin{array}{r}110 \\
75\end{array}$ & $\begin{array}{l}75 \\
47\end{array}$ & $\begin{array}{l}34 \\
28\end{array}$ & $\begin{array}{l}4.4 \\
4.5\end{array}$ & $\begin{array}{l}3.82 \\
3.73\end{array}$ & $\begin{array}{l}91 \\
93\end{array}$ & $\begin{array}{l}32 / 10 \\
32 / 14\end{array}$ & $\begin{array}{l}21 \\
24\end{array}$ & $\begin{array}{l}127 / 73 \\
120 / 76\end{array}$ & $\begin{array}{l}91 \\
92\end{array}$ \\
\hline
\end{tabular}


TABLE II.-Continued

\begin{tabular}{|c|c|c|c|c|c|c|c|c|c|c|c|}
\hline \multirow[t]{2}{*}{ Case } & \multirow{2}{*}{$\begin{array}{l}\text { Concen- } \\
\text { tration } \\
\text { inspired } \\
\text { oxygen } \\
\text { volumes \% }\end{array}$} & \multirow{2}{*}{$\begin{array}{c}\text { Alveolar } \\
\mathrm{pO}_{\mathbf{2}} \\
\boldsymbol{m} \boldsymbol{m} . \boldsymbol{H}_{\boldsymbol{g}}\end{array}$} & \multirow{2}{*}{$\begin{array}{c}\text { Arterial } \\
\mathrm{pO}_{2} \\
\mathbf{m} \boldsymbol{m} . \mathrm{Hg}_{\boldsymbol{g}}\end{array}$} & \multirow{2}{*}{$\begin{array}{c}\mathrm{MVB} \\
\mathrm{pO}_{2} \\
m m . \mathrm{Hg}_{\boldsymbol{g}}\end{array}$} & \multirow{2}{*}{$\begin{array}{c}\text { Arterio- } \\
\text { venous } \\
\text { oxygen diff.* } \\
\text { volumes \% }\end{array}$} & \multirow{2}{*}{$\begin{array}{c}\text { Cardiac } \\
\text { index } \\
\text { lit. } / \mathrm{min}_{\mathrm{BSA}} / \mathrm{m}^{2} \\
\mathrm{BSA}\end{array}$} & \multirow{2}{*}{$\begin{array}{l}\text { Heart rate } \\
\text { beats } / \text { min. }\end{array}$} & \multicolumn{2}{|c|}{$\begin{array}{c}\text { Pulmonary artery } \\
\text { pressure } \\
\text { mm. } \mathbf{H g}_{\mathbf{g}}\end{array}$} & \multicolumn{2}{|c|}{$\begin{array}{c}\text { Brachial artery } \\
\text { pressure } \\
\text { mm. } \mathbf{H g}_{\mathbf{g}}\end{array}$} \\
\hline & & & & & & & & $s / d$ & $m$ & $s / d$ & $m$ \\
\hline
\end{tabular}

(a) Normal and low oxygen-Continued

\begin{tabular}{l|l|l|l|l|l|l|l|l|l|l|l|l}
\hline 21 & 21.5 & 105 & 88 & 34 & 3.7 & 3.97 & 102 & $17 / 7$ & 13 & $124 / 76$ & 93 \\
& 16 & 69 & 66 & 30 & 3.1 & 4.50 & 102 & $14 / 7$ & 11 & $127 / 77$ & 93 \\
\hline
\end{tabular}

(b) Normal, low, and high oxygen

\begin{tabular}{|c|c|c|c|c|c|c|c|c|c|c|c|}
\hline 22 & $\begin{array}{l}21 \\
16 \\
21 \\
25\end{array}$ & $\begin{array}{r}94 \\
69 \\
110 \\
141\end{array}$ & $\begin{array}{l}62 \\
55 \\
60 \\
71\end{array}$ & $\begin{array}{l}36 \\
32 \\
37 \\
38\end{array}$ & $\begin{array}{l}4.1 \\
4.1 \\
4.6 \\
4.8\end{array}$ & $\begin{array}{l}3.33 \\
3.70 \\
3.43 \\
3.20\end{array}$ & $\begin{array}{r}90 \\
103 \\
93 \\
93\end{array}$ & $\begin{array}{l}26 / 11 \\
49 / 20 \\
39 / 18 \\
31 / 17\end{array}$ & $\begin{array}{l}15 \\
32 \\
27 \\
22\end{array}$ & $\begin{array}{l}109 / 62 \\
119 / 74 \\
142 / 83 \\
119 / 76\end{array}$ & $\begin{array}{r}80 \\
92 \\
104 \\
94\end{array}$ \\
\hline 23 & $\begin{array}{l}21.5 \\
16 \\
21 \dagger \\
30 \dagger\end{array}$ & $\begin{array}{r}101 \\
71 \\
104 \\
189\end{array}$ & $\begin{array}{r}60 \\
36 \\
61 \\
100\end{array}$ & $\begin{array}{l}22 \\
18 \\
29 \\
33\end{array}$ & $\begin{array}{l}3.9 \\
4.0 \\
4.7 \\
4.7\end{array}$ & $\begin{array}{l}3.62 \\
3.59 \\
2.71 \\
2.71\end{array}$ & $\begin{array}{l}95 \\
94 \\
68 \\
62\end{array}$ & $\begin{array}{l}35 / 17 \\
49 / 20 \\
36 / 9 \\
39 / 12\end{array}$ & $\begin{array}{l}24 \\
32 \\
19 \\
22\end{array}$ & $\begin{array}{l}102 / 63 \\
119 / 74 \\
121 / 61 \\
137 / 67\end{array}$ & $\begin{array}{l}79 \\
92 \\
85 \\
94\end{array}$ \\
\hline 24 & $\begin{array}{l}21 \\
16 \\
21 \\
25\end{array}$ & $\begin{array}{r}101 \\
72 \\
101 \\
129\end{array}$ & $\begin{array}{l}78 \\
63 \\
78 \\
87\end{array}$ & $\begin{array}{l}34 \\
32 \\
34 \\
36\end{array}$ & $\begin{array}{l}4.2 \\
3.7 \\
4.2 \\
5.1\end{array}$ & $\begin{array}{l}3.55 \\
3.86 \\
3.55 \\
2.71\end{array}$ & $\begin{array}{l}83 \\
88 \\
83 \\
83\end{array}$ & $\begin{array}{l}29 / 8 \\
35 / 9 \\
29 / 8 \\
31 / 7\end{array}$ & $\begin{array}{l}17 \\
19 \\
17 \\
17\end{array}$ & $\begin{array}{l}143 / 70 \\
142 / 72 \\
143 / 70 \\
153 / 74\end{array}$ & $\begin{array}{r}99 \\
100 \\
99 \\
104\end{array}$ \\
\hline 25 & $\begin{array}{l}21.5 \\
17.4 \\
21 \\
25\end{array}$ & $\begin{array}{r}118 \\
94 \\
112 \\
139\end{array}$ & $\begin{array}{l}84 \\
57 \\
84 \\
95\end{array}$ & $\begin{array}{l}28 \\
26 \\
28 \\
30\end{array}$ & $\begin{array}{l}5.1 \\
4.2 \\
5.1 \\
4.9\end{array}$ & $\begin{array}{l}3.07 \\
3.74 \\
3.07 \\
3.35\end{array}$ & $\begin{array}{l}87 \\
90 \\
90 \\
90\end{array}$ & $\begin{array}{l}59 / 27 \\
60 / 23 \\
59 / 27 \\
52 / 22\end{array}$ & $\begin{array}{l}37 \\
34 \\
37 \\
34\end{array}$ & $\begin{array}{l}170 / 105 \\
169 / 109 \\
183 / 112 \\
190 / 117\end{array}$ & $\begin{array}{l}131 \\
133 \\
135 \\
143\end{array}$ \\
\hline 26 & $\begin{array}{l}21.5 \\
16 \\
21 \\
30\end{array}$ & $\begin{array}{r}107 \\
66 \\
107 \\
167\end{array}$ & $\begin{array}{r}67 \\
40 \\
67 \\
110\end{array}$ & $\begin{array}{l}32 \\
29 \\
33 \\
40\end{array}$ & $\begin{array}{l}5.8 \\
4.8 \\
5.8 \\
5.6\end{array}$ & $\begin{array}{l}2.39 \\
2.88 \\
2.39 \\
2.41\end{array}$ & $\begin{array}{l}56 \\
66 \\
56 \\
60\end{array}$ & $\begin{array}{l}28 / 7 \\
32 / 11 \\
28 / 7 \\
30 / 9\end{array}$ & $\begin{array}{l}16 \\
20 \\
16 \\
17\end{array}$ & $\begin{array}{l}136 / 79 \\
128 / 77 \\
136 / 79 \\
141 / 80\end{array}$ & $\begin{array}{l}106 \\
101 \\
106 \\
102\end{array}$ \\
\hline 27 & $\begin{array}{l}21 \\
17.4 \\
21 \\
25\end{array}$ & $\begin{array}{r}103 \\
83 \\
103 \\
133\end{array}$ & $\begin{array}{l}63 \\
46 \\
63 \\
81\end{array}$ & $\begin{array}{l}19 \\
16 \\
20 \\
24\end{array}$ & $\begin{array}{l}5.9 \\
5.3 \\
5.9 \\
6.0\end{array}$ & $\begin{array}{l}3.45 \\
3.46 \\
3.45 \\
3.12\end{array}$ & $\begin{array}{r}100 \\
90 \\
100 \\
100\end{array}$ & $\begin{array}{l}50 / 23 \\
59 / 27 \\
50 / 23 \\
65 / 31\end{array}$ & $\begin{array}{l}34 \\
39 \\
34 \\
42\end{array}$ & $\begin{array}{r}91 / 65 \\
91 / 63 \\
91 / 65 \\
110 / 79\end{array}$ & $\begin{array}{l}77 \\
74 \\
77 \\
92\end{array}$ \\
\hline 28 & $\begin{array}{l}21 \\
17 \\
21 \\
25\end{array}$ & $\begin{array}{r}114 \\
82 \\
114 \\
142\end{array}$ & $\begin{array}{l}65 \\
40 \\
65 \\
77\end{array}$ & $\begin{array}{l}33 \\
31 \\
34 \\
37\end{array}$ & $\begin{array}{l}4.2 \\
3.2 \\
4.3 \\
4.0\end{array}$ & $\begin{array}{l}3.12 \\
3.94 \\
3.15 \\
3.14\end{array}$ & $\begin{array}{l}80 \\
80 \\
73 \\
76\end{array}$ & $\begin{array}{l}34 / 16 \\
48 / 23 \\
36 / 18 \\
34 / 17\end{array}$ & $\begin{array}{l}23 \\
33 \\
26 \\
24\end{array}$ & $\begin{array}{r}108 / 71 \\
105 / 71 \\
97 / 67 \\
99 / 66\end{array}$ & $\begin{array}{l}89 \\
87 \\
81 \\
81\end{array}$ \\
\hline
\end{tabular}

(c) Normal and high oxygen

\begin{tabular}{r|c|r|r|r|r|r|r|r|r|r|r}
\hline 29 & 21 & 95 & 54 & 37 & 3.5 & 5.20 & 137 & $42 / 19$ & 31 & $129 / 88$ & 108 \\
& 100 & - & 104 & 76 & 4.4 & 4.68 & 129 & $40 / 17$ & 28 & $131 / 88$ & 110 \\
30 & 21.5 & 88 & 48 & 30 & 4.5 & 3.62 & 75 & $42 / 18$ & 28 & $118 / 49$ & 75 \\
& 25 & 109 & 60 & 31 & 4.7 & 3.17 & 78 & $42 / 18$ & 27 & $124 / 50$ & 76 \\
31 & 21.5 & 116 & 64 & 34 & 4.0 & 3.53 & 76 & $22 / 7$ & 12 & $140 / 82$ & 108 \\
& 25 & 139 & 75 & 34 & 3.9 & 3.35 & 74 & $24 / 8$ & 15 & $146 / 88$ & 114 \\
& 33 & 190 & 83 & 34 & 3.9 & 3.52 & 73 & $20 / 6$ & 11 & $138 / 82$ & 105 \\
32 & 21 & 73 & 46 & 33 & 3.4 & 4.63 & 106 & $43 / 21$ & 30 & $112 / 72$ & 84 \\
& 25 & 102 & 75 & 38 & 3.4 & 4.89 & 114 & $51 / 24$ & 38 & $139 / 86$ & 105 \\
33 & 21 & 72 & 33 & 21 & 5.6 & 3.00 & 115 & $74 / 32$ & 46 & $121 / 84$ & 99 \\
& 25 & 102 & 50 & 28 & 5.6 & 2.88 & 108 & $77 / 36$ & 51 & $114 / 78$ & 92 \\
34 & 21 & 98 & 82 & 34 & 3.9 & 3.24 & 91 & $58 / 20$ & 34 & $121 / 69$ & 91 \\
& 100 & - & 100 & 111 & 4.3 & 2.83 & 85 & - & 31 & $127 / 69$ & 94 \\
35 & 21 & 105 & 45 & 26 & 5.6 & 2.57 & 88 & $67 / 26$ & 41 & $120 / 59$ & 84 \\
& 30 & 194 & 84 & 33 & 5.7 & 2.46 & 88 & $60 / 28$ & 40 & $125 / 62$ & 89 \\
\hline
\end{tabular}

* Using mixed venous blood obtained from the pulmonary artery.

$\dagger$ These observations were made on a second day. 


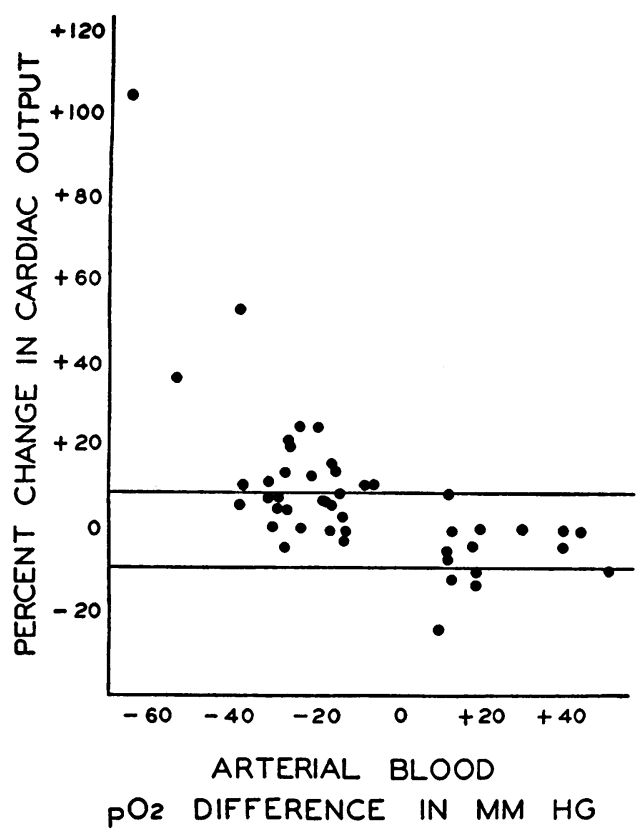

Fig. 1A. Relation Between Changes in Arterial Oxygen Tension and Cardiac Output

The cardiac output change is expressed in per cent of change from the initial measurement. A change of 9 per cent (above and below the two solid lines) is significant.

Note that during anoxia, cardiac output remains unchanged or rises and that increased oxygenation causes no change or a decrease in cardiac output.

insignificant change in heart rate (Table II). A persistent tachycardia was observed in seven of 28 patients during exposure to low oxygen.

\section{Cardiac output}

There was considerable variation in the level of the initial cardiac index in these experiments $(28$ normal $\left[3.09 \pm 0.5 \mathrm{lit} . / \mathrm{min} . / \mathrm{m}^{2}\right.$ B.S.], five low and 12 greater than normal). All 12 with high resting cardiac output had advanced pulmonary disease. Three (Cases 23, 29, 32) were in right heart failure; one (Case 1) had an associated interventricular septal defect. The effects of variation in level of oxygenation on cardiac output are illustrated in Figures 1 and 2. It is apparent that during exposure to low oxygen, the cardiac output either failed to change significantly (less than \pm 9 per cent) or increased. There was a significant increase in 14 of the 32 cases. In no instance was there a significant decrease. In 10 additional cases there were small increases, but less than the error of the method. The net experience suggests at least a tendency toward increased cardiac output during moderate anoxia in this series of cases.

On the other hand when we applied the Fick principle to a group of patients with similar clinical diagnoses, but which had been rejected from this study because, according to our criteria, they had failed to achieve a "steady state," the calculated cardiac outputs were found to vary greatly, often with values considerably below control. Since values for cardiac output so obtained are meaningless, we have not tabulated them.

Tables II and III further demonstrate that exposure to high oxygen in the steady state either effected no significant change or a decrease in cardiac output. The degree of change in cardiac output could not be quantitatively correlated with the concentration of oxygen in the inspired gas mixture. This is to be anticipated in the presence of chronic pulmonary disease, particularly in patients with alteration of the alveolar-capillary membrane (for instance Case 3), where decrease in inspired oxygen concentration will cause a greater decrease in arterial oxygen saturation than in normal subjects. The greatest changes in cardiac output were associated with marked changes in

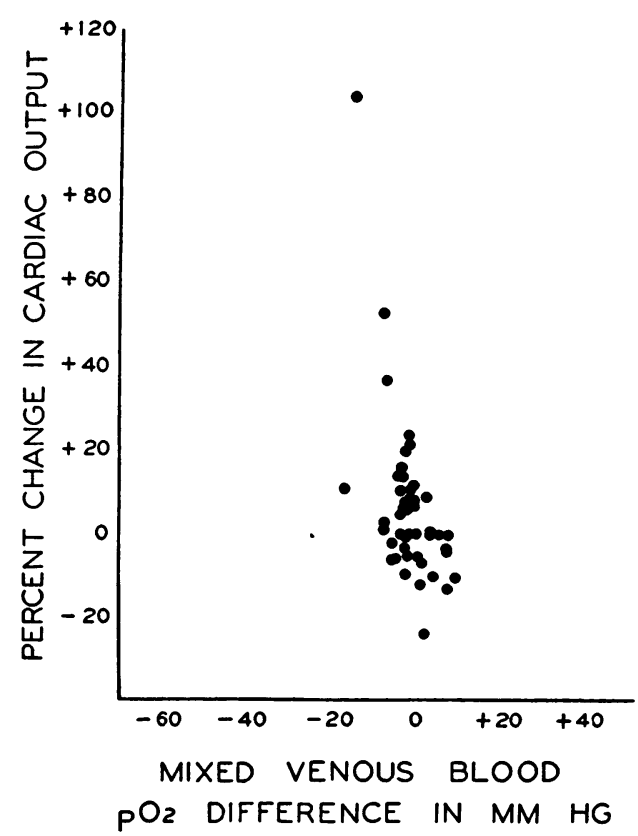

Fig. 1b. Relation Between Changes in Mixed Venous Blood Oxygen Tension and Cardiac Output Note that mixed venous blood oxygen tension is not related to cardiac output. 
arterial oxygen tension (Figure 1a). There was no significant relation between mixed venous oxygen tensions and cardiac output (Figure 1b).

\section{E. Pulmonary artery pressure (Table II)}

Following increase in the concentration of inspired oxygen, there was no significant change in mean pulmonary artery pressure, except in two subjects (cases 27 and 32), where the mean pulmonary artery pressure rose by $8 \mathrm{~mm}$. $\mathrm{Hg}$.

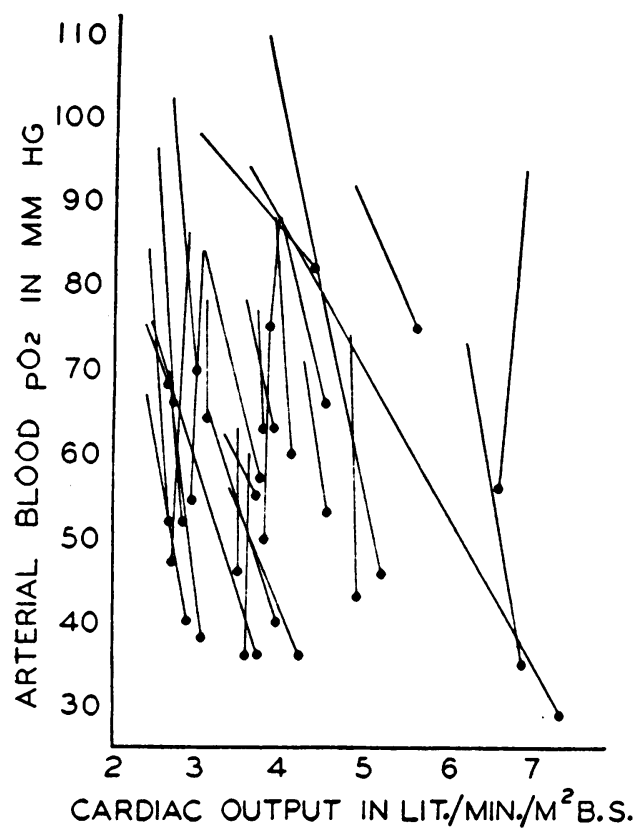

Fig. 2a. The Response of Cardiac Output to Acute Anoxia in Individual Patients

The upper end of each line indicates the arterial oxygen tension on 21 per cent oxygen; the lower knobbed end indicates the arterial oxygen tension during anoxia.

Note that the slope of the lines indicates that the cardiac output increased or did not change significantly during anoxia.

During low oxygen, mean pulmonary artery pressure rose more than $5 \mathrm{~mm}$. $\mathrm{Hg}$ in 10 of 38 experiments. Of the remaining 28 observations, 11 had increases in pulmonary artery pressure, during anoxia, of 3 to $5 \mathrm{~mm}$. $\mathrm{Hg}$. Not all subjects with increased pressures during anoxia had increased cardiac outputs. However, the subjects (Cases 4 and 22) with the largest increases in pulmonary artery pressure had significant simultaneous increases in cardiac output.

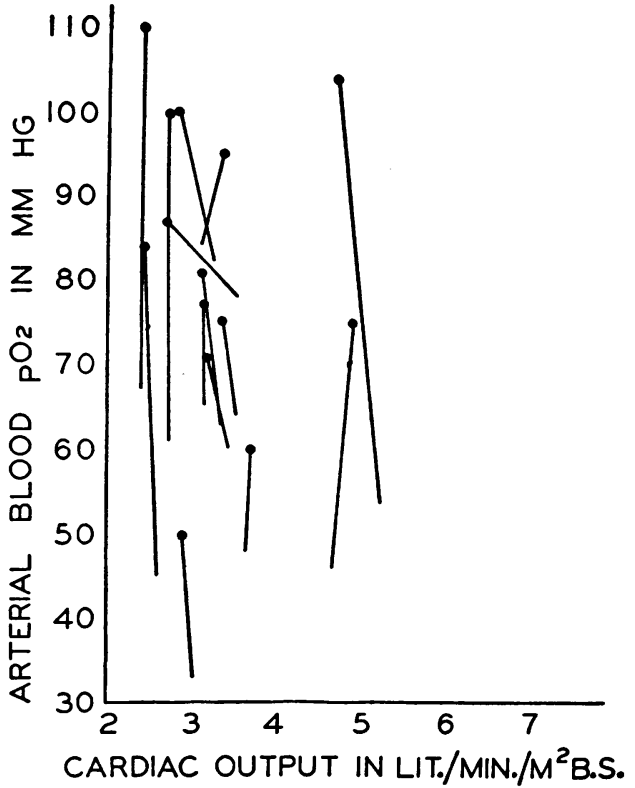

Fig. 2B. The Response of Caruiac Output to InCREase in ARterial Oxygen Tension in INdividual Patients

The lower end of each line indicates the arterial oxygen tension on 21 per cent oxygen; the upper knobbed end indicates the arterial oxygen tension at the higher level of oxygenation.

Note that at these higher levels of arterial oxygen tension there was either no significant change or a fall in cardiac output.

\section{F. Brachial artery pressure}

The changes in brachial artery pressure following high and low oxygen showed no consistent trend. Only one subject (Case 32) underwent a large change in mean pressure $(+21$ per cent) while breathing high oxygen.

\section{DISCUSSION}

The importance of achieving the "steady state" when one determines cardiac output by the Fick principle $^{4}$ has been emphasized in this presenta-

4 The Fick principle states that the quantity of any substance taken up by blood perfusing an organ is equal to the amount of that substance in the volume of blood leaving the organ minus the amount contained in the volume of blood entering the organ. For the instantaneous uptake of oxygen by blood in the lungs

$$
\dot{\mathrm{V}}_{\mathrm{O}_{3}}=\frac{\mathrm{dV} \mathrm{O}_{2}}{\mathrm{dt}}=\left[\mathrm{CaO}_{3}(\mathrm{t})-\mathrm{Cv}_{\mathrm{O}_{2}}(\mathrm{t})\right] Q(\mathrm{t}) \text {, Equation } 1
$$

where $Q=$ instantaneous blood flow to lungs $=$ instantaneous blood flow from lungs at time $(t)$ and $\mathrm{Ca}_{2}$, $\mathrm{CvO}_{3}=$ instantaneous concentrations of oxygen in arterial 
tion. Failure to recognize the importance of this factor may explain some of the discrepancies in previous observations on the effects of anoxia upon the circulation. The difficulty in achieving a "steady state," even in normal subjects exposed to moderate anoxia, is clearly indicated by the data of Rahn and Otis (15). In their studies, normal trained subjects were acutely elevated to simulated altitude, and the time was noted for restoration of heart rate, R.Q., and oxygen consumption to normal. They found that the higher the altitude, the longer the time required for reequilibration, and at 10 per cent oxygen (roughly and mixed venous blood respectively, at time $(t)$, from which,

$$
Q(t)=\frac{\dot{\mathrm{V}}_{\mathrm{O}_{2}}}{\mathrm{Ca}_{\mathrm{O}_{2}}-\mathrm{Cv}_{\mathrm{O}_{2}}} \quad \text { Equation } 2
$$

and generalizing, the mean flow $(\bar{Q})$ during time $(t)$, becomes

$$
\overline{\mathrm{Q}}=\frac{1}{\mathrm{~T}} \int_{0}^{\mathrm{T}} \frac{\dot{\mathrm{V}}_{\mathrm{O}_{2}}}{\mathrm{Ca}_{\mathrm{O}_{2}}-\mathrm{CV}_{\mathrm{O}_{2}}} \mathrm{dt} \text {. Equation } 3
$$

When the quantities $\mathrm{V}_{\mathrm{O}_{2}}, \mathrm{Ca}_{\mathrm{O}_{2}}-\mathrm{CV}_{\mathrm{O}_{2}}$ remain constant (or do not significantly differ from the mean), then,

$$
\overline{\mathrm{Q}}=\frac{\overline{\mathrm{V}}_{\mathrm{O}_{2}}}{\overline{\mathrm{Ca}_{\mathrm{O}_{2}}-\mathrm{CVO}_{2}}} \cdot \quad \text { Equation } 4
$$

In this equation $\overline{\mathrm{Q}}, \overline{\mathrm{V}}_{\mathrm{O}_{2}}$, and $\overline{\mathrm{Ca}_{2}-\mathrm{Cv}_{2}}$ represent mean values during the time $t$. This is the form of the Fick principle generally used for calculation of cardiac output. Implicit to its application are the concepts that oxygen is neither secreted nor stored in the lungs.

For substitution in Equation 4, it is assumed that the oxygen uptake, as measured from collection and analysis of expired gas, is equal to the oxygen taken up by the blood in its passage through the lungs; which in turn is equal to the oxygen consumed by the tissues. This relationship does not obtain during the period immediately following the change-over from ambient air to the anoxic mixture. During this period of adjustment to the acute anoxia, the quantity of oxygen in the blood phase and the gas phase is greater than will exist when equilibrium is achieved. Consequently tissue need for oxygen will be met not only from the inspired low oxygen mixture, but also from the surplus oxygen retained in the circulating blood and lungs from the previous level of oxygenation. The oxygen consumption as measured from the spirometer ("apparent oxygen consumption") will thus be lower than both the actual tissue oxygen consumption and the oxygen taken up by the blood traversing the lungs. Cardiac output calculated from this "apparent oxygen consumption" will be lower than the true cardiac output.

Another difficulty in the unsteady state is to relate the measured oxygen uptake to the blood samples responsible for its uptake. Simultaneous and prolonged collection periods for blood and gas do not completely solve this problem, since the proper time relationship is unpredictable. equivalent to 18,000 feet) an hour or more was required for these measurements to return to control levels. It is apparent that if our patients had been exposed to these levels of anoxia, a steady state would have been difficult or impossible to approach during the time (15 minutes or more) allotted for equilibration. This is emphasized by the large number of patients who were excluded from this presentation because of their inability to reach a "steady state" even with mild depression of inspired oxygen content.

In our subjects presented above, despite the presence of advanced lung disease, it was possible to approximate a "steady state" by careful selection of the inspired oxygen mixture so as to avoid extreme variations in arterial oxygen saturation. Under these conditions, no significant fall in cardiac output was ever observed during anoxia. This finding led us to re-evaluate the observations on cardiac output previously recorded from this laboratory (3) incident to a study of changes in pulmonary artery pressure in five normal subjects who were exposed to low oxygen mixtures (10 per cent) for brief periods (about 10 minutes). The average cardiac output of the five subjects was reported to have decreased from 5.74 liters to 5.20 liters. A review of the original data from which these results were calculated indicated that these subjects were not in a "steady state" (high initial R.Q., marked variations in successive R.Q. values, and unusually low oxygen intake at the low level of oxygenation). Similar considerations apply to the fall in cardiac output during acute anoxia recently reported from another laboratory (4). Such evidence of an unsteady state invalidates the use of the Fick principle for calculation of cardiac output.

The need for a "steady state" prior to the application of the Fick principle obviously extends to conditions other than acute anoxia, e.g., exercise. Bock, Dill, and their associates (17) stressed the difficulties in reaching a "steady state" during exercise, and urged caution in the interpretation of measurements so made. The evaluation of measurements of cardiac output after exercising patients with heart disease for three minutes is consequently difficult (18).

The other recorded changes in the circulation in man are generally in accord with previous data 
TABLE III

Summary of changes in circulation and respiration at two levels of oxygenation

\begin{tabular}{|c|c|c|c|c|c|c|c|c|c|c|}
\hline Case* & $\begin{array}{l}\text { Final con- } \\
\text { centration } \\
\text { inspired } \\
\text { oxygent } \\
\%\end{array}$ & $\underset{\%}{\text { Ventilation }}$ & $\underset{\substack{\text { Oxygen } \\
\text { consump- } \\
\text { tion } \\
\%}}{ }$ & R.Q.f & $\begin{array}{c}\mathrm{pO}_{2} \\
\text { arterial } \\
\text { bloodf } \\
\text { mm. } \mathrm{H}_{8}\end{array}$ & $\underset{m m . \mathrm{Hg}_{\mathbf{g}}}{\mathrm{PO}_{\mathbf{2}}}$ & $\begin{array}{c}\text { Heart } \\
\text { rate } \neq \\
\text { beats } / \text { min. }\end{array}$ & $\begin{array}{c}\text { Cardiac } \\
\text { index } \\
\%\end{array}$ & $\begin{array}{c}\text { Pulmonary } \\
\text { artery } \\
\text { pressure } \\
\text { mean } \\
\text { mm. } \text { Hg }_{\mathbf{g}}\end{array}$ & $\begin{array}{c}\text { Brachial } \\
\text { artery } \\
\text { pressuref } \\
\text { mear } \\
\text { mm. } \mathrm{H}_{\mathbf{g}}\end{array}$ \\
\hline
\end{tabular}

(a) From normal to low oxygen

\begin{tabular}{|c|c|c|c|c|c|c|c|c|c|c|}
\hline 1 & 10 & +25.8 & +11 & -.05 & -38 & -17 & +2 & +11 & -2 & -13 \\
\hline 2 & 12 & +41.2 & +1.5 & +.11 & -54 & -7 & +13 & +37 & +2 & -9 \\
\hline 3 & 14 & -5.9 & 0 & 0 & -65 & -15 & +19 & +104 & +7 & +10 \\
\hline 4 & 12 & +36.9 & +11 & +.04 & -39 & -8 & +13 & +53 & +15 & +14 \\
\hline 5 & 18.5 & +11.4 & +6.8 & +.06 & -9 & -2 & +7 & +11 & 0 & 0 \\
\hline 6 & $\begin{array}{l}16 \\
16\end{array}$ & $\begin{array}{l}-4.2 \\
+27\end{array}$ & $\begin{array}{r}8.6 \\
+0.5\end{array}$ & $\begin{array}{l}+.11 \\
-.02\end{array}$ & $\begin{array}{l}-32 \\
-32\end{array}$ & $\begin{array}{l}-1 \\
-3\end{array}$ & $\begin{array}{l}+3 \\
+6\end{array}$ & $\begin{array}{l}+12 \\
+\quad 8\end{array}$ & +3 & +6 \\
\hline 7 & 16 & +9.2 & +9 & 0 & -14 & -8 & -3 & +3 & -1 & -5 \\
\hline 8 & $\begin{array}{l}16 \\
16\end{array}$ & $\begin{array}{r}+8.3 \\
-7.9\end{array}$ & $\begin{array}{r}3 \\
0\end{array}$ & $\begin{array}{l}-.03 \\
+.01\end{array}$ & $\begin{array}{l}-31 \\
-28\end{array}$ & $\begin{array}{l}-8 \\
-6\end{array}$ & $\begin{array}{r}+8 \\
+10\end{array}$ & $\begin{array}{l} \pm \quad 1 \\
-\quad 6\end{array}$ & $\begin{array}{r}+5 \\
+4\end{array}$ & $\begin{array}{l}-9 \\
-8\end{array}$ \\
\hline 9 & $\begin{array}{l}16 \\
14\end{array}$ & $\begin{array}{l}-8.6 \\
-12.4\end{array}$ & $\begin{array}{l}+4.4 \\
+1.5\end{array}$ & $\begin{array}{l}+.04 \\
+.03\end{array}$ & $\begin{array}{l}-19 \\
-28\end{array}$ & $\begin{array}{l}-3 \\
-4\end{array}$ & $\begin{array}{l}+3 \\
+6\end{array}$ & $\begin{array}{r}+7 \\
+14\end{array}$ & $\begin{array}{r}+3 \\
+7\end{array}$ & $\begin{array}{l}-4 \\
+3\end{array}$ \\
\hline 10 & $\begin{array}{l}17 \\
17\end{array}$ & $\begin{array}{r}+10.7 \\
+7.9\end{array}$ & $\begin{array}{l}-3.4 \\
-5.1\end{array}$ & $\begin{array}{l}+.09 \\
+.06\end{array}$ & $\begin{array}{l}-20 \\
-18\end{array}$ & $\begin{array}{l}-2 \\
-3\end{array}$ & $\begin{array}{r}0 \\
-3\end{array}$ & $\begin{array}{l}24 \\
+\quad 7\end{array}$ & $\begin{array}{r}+6 \\
+10\end{array}$ & 0 \\
\hline 11 & 16 & +8.3 & -7.5 & +.08 & -14 & -3 & 0 & & +1 & +4 \\
\hline 12 & 16 & 0 & -6.2 & +.05 & -28 & -4 & +10 & +5 & +4 & +1 \\
\hline 13 & 16 & -3.6 & -4.0 & +.06 & -17 & -3 & 0 & +6 & +2 & -2 \\
\hline 14 & 16 & +2.7 & -7.2 & +.08 & -30 & -2 & +14 & +8 & +5 & -3 \\
\hline 15 & 16 & +40.0 & 0 & +.11 & -17 & -4 & +4 & +16 & +1 & -3 \\
\hline 16 & 16 & 0 & 0 & -.02 & -30 & -2 & 0 & -5 & +3 & -6 \\
\hline 17 & 16 & +19.0 & -6.4 & +.10 & -39 & -6 & -1 & -6 & +4 & -4 \\
\hline 18 & 16 & +9.3 & 0 & +.09 & -14 & -6 & -2 & -3 & +7 & -8 \\
\hline 19 & 16 & +22.5 & +5.8 & +.09 & -16 & -2 & +8 & +14 & +1 & -1 \\
\hline 20 & 16 & +12.8 & 0 & +.01 & -28 & -6 & +2 & -2 & +3 & +1 \\
\hline 21 & 16 & +3.6 & -4.8 & +.07 & -22 & -4 & 0 & +13 & -2 & 0 \\
\hline
\end{tabular}

from this laboratory (3), and, in animals, from other laboratories (19). Motley and his associates (3) found that pulmonary artery pressure uniformly increases in normal subjects acutely exposed to 10 per cent oxygen. Approximately onethird of our patients with pulmonary disease manifested a similar response to anoxia. However, the failure of 28 of the 38 to have a significant increase in pulmonary artery pressure at the end of 15 to 20 minutes of anoxia deserves comment. At least three other factors may be considered in evaluating this finding: 1) The observed tendency of the elevated pulmonary artery pressures to fall towards initial levels in many of these patients as breathing at the low level of oxygenation is continued; 2) the difference which may exist between the response of the chronically anoxic (acclimatized) and normal subject to acute anoxia; and 3) the significantly higher $\mathrm{pO}_{2}$ alveolar level attained in these studies when compared to the previous studies in normal animals and man $(3,19)$.

Whether a single mechanism is involved in the 
TABLE III-Continued

\begin{tabular}{|c|c|c|c|c|c|c|c|c|c|c|}
\hline Case* & $\begin{array}{c}\text { Final con- } \\
\text { centration } \\
\text { inspired } \\
\text { oxygent } \\
\%\end{array}$ & $\begin{array}{c}\text { Ventilation } \neq \\
\%\end{array}$ & $\underset{\substack{\text { Oxygen } \\
\text { consump- } \\
\%}}{\%}$ & R.Q.‡ & $\begin{array}{c}\mathrm{pO}_{2} \\
\text { arterial } \\
\text { bloodf } \\
\text { mm. } \boldsymbol{H}_{\boldsymbol{g}}\end{array}$ & $\underset{m m . \mathrm{Hg}_{\mathrm{g}}}{\mathrm{PO}_{2}}$ & $\begin{array}{c}\text { Heart } \\
\text { ratet } \\
\text { beats } / \text { min. }\end{array}$ & 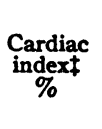 & $\begin{array}{c}\text { Pulmonary } \\
\text { artery } \\
\text { pressureł } \\
\text { mean } \\
\text { mm. } \boldsymbol{B g}_{\boldsymbol{g}}\end{array}$ & $\begin{array}{c}\text { Brachial } \\
\text { artery } \\
\text { pressuret } \\
\text { mean } \\
\text { mm. Hg }\end{array}$ \\
\hline
\end{tabular}

(b) From normal to low and to high oxygen

\begin{tabular}{|c|c|c|c|c|c|c|c|c|c|c|}
\hline 22 & $\begin{array}{l}16 \\
25\end{array}$ & $\begin{array}{l}+9.1 \\
-8\end{array}$ & $\begin{array}{l}+11 \\
-2\end{array}$ & $\begin{array}{l}+.06 \\
-.11\end{array}$ & $\begin{array}{r}-7 \\
+11\end{array}$ & $\begin{array}{l}-4 \\
+1\end{array}$ & $\begin{array}{r}+13 \\
0\end{array}$ & $\begin{array}{l}+11 \\
-\quad 7\end{array}$ & $\begin{array}{l}+17 \\
-5\end{array}$ & $\begin{array}{l}+12 \\
-10\end{array}$ \\
\hline 23 & $\begin{array}{l}16 \\
30\end{array}$ & $\begin{array}{r}+8.3 \\
+9.3\end{array}$ & $\begin{array}{r}2 \\
0\end{array}$ & $\begin{array}{l}+.09 \\
+.09\end{array}$ & $\begin{array}{l}-24 \\
+39\end{array}$ & $\begin{array}{l}-4 \\
+4\end{array}$ & $\begin{array}{l}-1 \\
-6\end{array}$ & $\begin{array}{l}0 \\
0\end{array}$ & $\begin{array}{l}+8 \\
+3\end{array}$ & $\begin{array}{l}+13 \\
+9\end{array}$ \\
\hline 24 & $\begin{array}{l}16 \\
25\end{array}$ & $\begin{array}{l}-14.0 \\
+7\end{array}$ & $\begin{array}{r}+0.5 \\
+7.5\end{array}$ & $\begin{array}{l}+.08 \\
+.03\end{array}$ & $\begin{array}{l}-15 \\
+9\end{array}$ & $\begin{array}{l}-2 \\
+2\end{array}$ & $+\begin{array}{l}5 \\
0\end{array}$ & $\begin{array}{l}+9 \\
-24\end{array}$ & $\begin{array}{r}2 \\
0\end{array}$ & +5 \\
\hline 25 & $\begin{array}{l}17.4 \\
25\end{array}$ & $\begin{array}{r}8.1 \\
+12.1\end{array}$ & $\begin{array}{l}0 \\
+\quad 5.8\end{array}$ & $\begin{array}{l}+.04 \\
+.06\end{array}$ & $\begin{array}{l}-27 \\
+11\end{array}$ & $\begin{array}{l}-2 \\
+\quad 2\end{array}$ & $\begin{array}{r}3 \\
0\end{array}$ & $\begin{array}{l}+22 \\
+\quad 9\end{array}$ & $\begin{array}{l}-3 \\
-3\end{array}$ & $\begin{array}{l}+2 \\
+8\end{array}$ \\
\hline 26 & $\begin{array}{l}16 \\
30\end{array}$ & $\begin{array}{l}+3.5 \\
-6.9\end{array}$ & $\begin{array}{l}0 \\
-\quad 2.2\end{array}$ & $\begin{array}{l}+.02 \\
-.02\end{array}$ & $\begin{array}{l}-27 \\
+43\end{array}$ & $\begin{array}{l}-3 \\
+7\end{array}$ & $\begin{array}{l}+10 \\
+4\end{array}$ & $\begin{array}{r}20 \\
0\end{array}$ & $\begin{array}{l}+4 \\
+1\end{array}$ & $\begin{array}{l}-5 \\
-4\end{array}$ \\
\hline 27 & $\begin{array}{l}17.4 \\
25\end{array}$ & $\begin{array}{l}0 \\
0\end{array}$ & $\begin{array}{l}-9.3 \\
-8.8\end{array}$ & $\begin{array}{l}+.06 \\
+.05\end{array}$ & $\begin{array}{r}-17 \\
+18\end{array}$ & $\begin{array}{l}-3 \\
+4\end{array}$ & $\begin{array}{r}-10 \\
0\end{array}$ & $\begin{array}{r}0 \\
-\quad 10\end{array}$ & $\begin{array}{l}+5 \\
+8\end{array}$ & $\begin{array}{r}-3 \\
+15\end{array}$ \\
\hline 28 & $\begin{array}{l}17 \\
25\end{array}$ & $\begin{array}{l}0 \\
+1.6\end{array}$ & $\begin{array}{l}-4.1 \\
-7.9\end{array}$ & $\begin{array}{l}+.07 \\
+.10\end{array}$ & $\begin{array}{l}-25 \\
+12\end{array}$ & $\begin{array}{l}-2 \\
+3\end{array}$ & $\begin{array}{r}0 \\
+3\end{array}$ & $\begin{array}{r}25 \\
0\end{array}$ & $\begin{array}{l}+10 \\
-2\end{array}$ & $\begin{array}{r}-2 \\
0\end{array}$ \\
\hline
\end{tabular}

(c) From normal to high oxygen

\begin{tabular}{r|r|r|r|r|r|r|r|r|r|r}
\hline 29 & 100 & & +11 & & +50 & +9 & -8 & -10 & -3 & +2 \\
30 & 25 & +12 & -6 & -.01 & +12 & +1 & +3 & -12 & +3 & +11 \\
31 & 25 & 0 & -8 & +.11 & +11 & 0 & -2 & -5 & -3 & +6 \\
& 33 & 0 & -3 & +.06 & +19 & 0 & -3 & 0 & -1 & -3 \\
32 & 25 & +1 & +5 & -.05 & +29 & +5 & +8 & 0 & +8 & +21 \\
33 & 25 & -1 & -4 & -.03 & +17 & +7 & +3 & -4 & +5 & -7 \\
34 & 100 & & -4 & & +18 & +7 & -6 & -13 & -3 & +3 \\
35 & 30 & -7.0 & -2.7 & -.02 & +39 & +7 & 0 & -4 & -1 & +5 \\
\hline
\end{tabular}

* As in Tables I and II.

+ Second level of oxygenation. Initial level in all instances was ambient or compressed air.

$\ddagger$ As compared to study with ambient or compressed air.

cases in whom the pulmonary artery pressure increased during anoxia is not clear. Indeed, our data do not provide any information concerning the contribution of the pulmonary veins or left auricle to the elevation in the pulmonary arterial pressure. However, in this group of patients with chronic pulmonary disease, an increase of blood flow in a pathologically restricted vascular bed may cause a rise in pulmonary arterial pressure. This factor could be invoked in seven of the 10 subjects in whom an increase in pulmonary arterial pressure was observed following low oxygen breathing.

Changes in cardiac output greater than 15 per cent at the lower level of oxygenation occurred in nine of the 47 determinations. Such marked changes are of particular importance in the method which Riley, Cournand and Donald (6) have recently described for estimating the oxygen-diffusing capacity and the ventilation-perfusion relationships of the lung. Our data suggest that the assumption of a relatively constant cardiac output in subjects exposed to two levels of inspired oxygen, an assumption which is essential to their method, is most apt to be valid if extreme variation in arterial oxygen tension is avoided by careful selection of inspired oxygen mixtures and the use of an oximeter. However, even with this precau- 
tion, a significant, but at the present time unpredictable, increase in cardiac output may occur in some patients with pulmonary disease.

\section{SUM MARY}

1. The circulatory responses of 35 patients with pulmonary and cardiopulmonary disease to selected levels of oxygenation (higher and lower than room air) were investigated.

2. Particular care was exerted to arrive at a "steady state" of the respiration and circulation. The criteria for the "steady state" are defined; the relation of the "steady state" to the applicability of the Fick principle for cardiac output measurement is discussed.

3. After the "steady state" was achieved in the patients with chronic pulmonary disease exposed to moderate anoxia, cardiac output remained unchanged or increased. Conversely, an increase in concentration of inspired oxygen caused either no change or occasionally a slight fall in cardiac output. The largest increases in cardiac output during anoxia were associated with marked decreases in arterial oxygen pressure.

4. In response to anoxia, pulmonary artery pressure increased significantly (more than $5 \mathrm{~mm} . \mathrm{Hg}$ ) in a third of these patients with pulmonary disease and remained unchanged in the others.

\section{ACKNOWLEDGMENT}

The authors gratefully acknowledge the aid which they received from Professor Georges A. Deschamps in the preparation of the footnote.

\section{REFERENCES}

1. Grollman, A., The Cardiac Output of Man in Health and Disease. Charles C Thomas Co., Springfield, 1932.

2. Harrison, T. R., Wilson, C. P., Neighbors, D. W., and Pilcher, C., The regulation of the circulation. VII. The effects of anoxemia of mild degree on the cardiac output of unnarcotized dogs. Am. J. Physiol., 1927-28, 83, 275.

3. Motley, H. L., Cournand, A., Werkö, L., Himmelstein, A., and Dresdale, D., The influence of short periods of induced acute anoxia upon pulmonary artery pressures in man. Am. J. Physiol., 1947, $150,315$.

4. Westcott, R. N., Fowler, N. O., Scott, R. C., Hauenstein, V. D., and McGuire, J., Anoxia and human pulmonary vascular resistance. J. Clin. Invest., 1951, 30, 957.
5. Lilienthal, J. L., Jr., Riley, R. L., Proemmel, D. D., and Franke, R. E., An experimental analysis in man of the oxygen pressure gradient from alveolar air to arterial blood during rest and exercise at sea level and at altitude. Am. J. Physiol., 1946, 147, 1919.

6. Riley, R. L., Cournand, A., and Donald, K. W., Analysis of factors affecting partial pressures of oxygen and carbon dioxide in gas and blood of lungs; methods. J. Appl. Physiol., 1951, 4, 102.

7. Riley, R. L., Proemmel, D. D., and Franke, R. E., A direct method for determination of oxygen and carbon dioxide tensions in blood. J. Biol. Chem., 1945, 161, 621.

8. Harvey, R. M., Ferrer, M. I., Cathcart, R. T., Richards, D. W., Jr., and Cournand, A., Some effects of digoxin upon the heart and circulation in man. Digoxin in left ventricular failure. Am. J. Med., 1949, 7, 439.

9. Ferrer, M. I., Harvey, R. M., Cathcart, R. T., Webster, C. A., Richards, D. W., Jr., and Cournand, A., Some effects of digoxin upon the heart and circulation in man. Digoxin in chronic cor pulmonale. Circulation, 1950, 1, 161.

10. Cournand, A., The Fourth Walter Wile Hamburger Memorial Lecture, Institute of Medicine of Chicago. Some aspects of the pulmonary circulation in normal man and in chronic cardiopulmonary diseases. Circulation, 1950, 2, 641.

11. Ziegler, L. H., and Levine, B. S., The influence of emotional reactions on basal metabolism. Am. J. M. Sc., 1925, 169, 68.

12. Benedict, F. G., Degree of constancy in human basal metabolism. Am. J. Physiol., 1934-35, 110, 521.

13. Du Bois, E. F., Basal Metabolism in Health and Disease. Lea \& Febiger, Philadelphia, 1936, 3rd ed.

14. Grollman, A., Physiological variations of the cardiac output of man. VII. The effects of high altitude on cardiac output and its related functions: an account of experiments conducted on the summit of Pikes Peak, Colorado. Am. J. Physiol., 1930, 93, 19.

15. Rahn, H., and Otis, A. B., Man's respiratory responses during and after acclimatization to high altitude. Am. J. Physiol., 1949, 157, 445.

16. Houston, C. S., and Riley, R. L., Respiratory and circulatory changes during acclimatization to high altitude. Am. J. Physiol., 1947, 149, 565.

17. Bock, A. V., Van Caulaert, C., Dill, D. B., Fölling, A., and Hurxthal, L. M., Studies in muscular activity. IV. The "steady state" and the respiratory quotient during work. J. Physiol., 1928, 66, 162.

18. Gorlin, R., Sawyer, C. G., Haynes, F. W., Goodale, W. T., and Dexter, L., Effects of exercise on circulatory dynamics in mitral stenosis. III. Am. Heart J., 1951, 41, 192.

19. v. Euler, U. S., and Liljestrand, G., Observations on the pulmonary arterial blood pressure in the cat. Acta physiol. Scandinav., 1946, 12, 301. 